\title{
Appréhender les outils simples en contexte préhistorique
}

Sophie A. de Beaune

\section{(2) OpenEdition}

Journals

Édition électronique

URL : https://journals.openedition.org/artefact/9110

DOI : $10.4000 /$ artefact.9110

ISSN : 2606-9245

Éditeur :

Association Artefact. Techniques histoire et sciences humaines, Presses universitaires du Midi

Édition imprimée

Date de publication : 11 septembre 2014

Pagination : 137-148

ISBN : 978-2-271-08150-6

ISSN : 2273-0753

Référence électronique

Sophie A. de Beaune, "Appréhender les outils simples en contexte préhistorique ", Artefact [En ligne], 2 | 2014, mis en ligne le 12 mai 2021, consulté le 24 août 2021. URL : http://journals.openedition.org/ artefact/9110; DOI : https://doi.org/10.4000/artefact.9110

\section{(c) (i) (9)}

Artefact, Techniques, histoire et sciences humaines est mise à disposition selon les termes de la Licence Creative Commons Attribution - Pas d'Utilisation Commerciale - Pas de Modification 4.0 International. 


\section{Appréhender les outils simples en contexte préhistorique}

Sophie A. DE BEAUNE

\section{Résumé}

L'auteur discute ici de la notion d'«outil simple» en contexte préhistorique: outils sans aménagement, utilisés bruts, ou bien outils «expédients», n'ayant servi que brièvement pour répondre à un besoin immédiat. Ces outils simples, qui ne sont pas nécessairement anciens, peuvent pourtant faire partie intégrante d'une chaîne opératoire complexe. L'auteur pose d'autres questions à leur sujet: sont-ils propres à l'homme? Comment peut-on les reconnaître en contexte archéologique? Leurs utilisateurs ont-ils un statut particulier?

Mots-clés: naturefacts, outillage non façonné, outils animaux, outils expédients, paléolithique.

\section{Abstract}

In this paper, the author discusses the notion of «simple tools» in a prehistoric context: Are they unworked and raw tools, or expedient tools, only used for a limited time to meet a specific need? These simple tools, which are not necessarily old, could, however, be part of a complex chain of operations. The author asks other questions about them: such as "Are they exclusive to humans?"; "How can we recognize them in an archaeological context?" and "Did their users have a specific rank or status?"

Keywords: animal tools, expedient technology, naturefacts, Palaeolithic, unworked tools. 
Les outils simples n'ont pas grandchose de commun entre eux, si ce n'est la simplicité, plus apparente que réelle, qui en a fait négliger l'étude. Et cette négligence ne touche pas que l'archéologie. François Sigaut, Haris Procopiou et moimême avons eu le sentiment qu'il y avait des trouvailles à faire dans ce recoin obscur des techniques et c'est pourquoi nous avons organisé plusieurs séances de séminaire sur le sujet, en faisant appel à des archéologues, des anthropologues et des historiens des techniques ${ }^{2}$.

Avant tout, il est apparu que la notion même de «simplicité » posait problème. Quels étaient donc les objets susceptibles d'être considérés comme «simples»? En fait, un outil n'est pas simple en soi, il l'est sous un certain rapport, et tout dépend donc du rapport sous lequel on l'envisage. On pourra considérer comme simple un outil dont le façonnage est sommaire ou inexistant. Ou bien un outil qui n'aura servi qu'une fois, ou du moins pendant une période de temps très brève. Ou bien un outil sera considéré comme simple par opposition à d'autres outils composés de plusieurs pièces assemblées. Dans le premier cas, c'est la fabrication de l'outil qui est simple, parce que réduite à rien ou presque; dans le deuxième, c'est sa biographie qui l'est, là encore parce que réduite à peu de chose ; dans le dernier cas, l'outil est simple par comparaison avec d'autres outils qui le sont moins. Ce sont surtout les deux premiers cas qui vont nous occuper, mais notons tout de même qu'il y a un rapport immédiat entre le premier et le troisième sens du mot «simple» cerné ici: un outil peu ou pas façonné est en particulier exempt de cette sorte particulière de façonnage qui consiste à assembler plusieurs éléments pour composer un tout. Nous verrons en revanche que le lien entre le premier et le deuxième sens $\mathrm{du}$ mot est plus complexe. Quelle que soit leur manière d'être simple, on peut s'interroger sur le statut de ces outils et se demander s'ils sont réservés à une certaine catégorie d'utilisateurs.

\section{Qu'est-ce qu'un outil simple?}

Les outils simples au premier sens sont des objets naturels utilisés bruts. Wendell $\mathrm{H}$. Oswalt ${ }^{3}$ a proposé le terme de naturefacts pour désigner ces objets, par opposition aux artefacts qui, eux, sont transformés. Les mots ici sont parlants: certains outils sont les produits de la nature, d'autres sont les produits de l'art. Ainsi, de simples galets utilisés pour écraser ou faire éclater des matières animales ou végétales, à l'instar des casse-noix encore utilisés aujourd'hui au
Maroc pour concasser les noix d'argane ${ }^{4}$, entrent parfaitement dans cette définition. On pourrait considérer alors que, pour l'observateur, ces outils sont finalement des outils a posteriori, puisqu'ils ne peuvent être vus comme tels qu'après leur usage.

Il est cependant raisonnable de considérer également comme simples des outils ne portant que quelques traces d'aménagement. Sans être des produits de la nature, ces outils n'ont nécessité 
que bien peu d'art. Ainsi, les meules et les broyeurs rudimentaires du Paléolithique supérieur ont parfois été légèrement piquetés sur leur surface pour en améliorer le mordant ${ }^{5}$.

Il faut préciser que, dans le cas de certains outils retrouvés en contexte archéologique, le caractère intentionnel de certaines traces n'est pas toujours facile à déterminer: le départ entre ce qui revient au façonnage et ce qui a été produit par l'usure n'est pas toujours clair. Il arrive en effet que la forme de ces outils ait été modifiée par l'usage et il peut être délicat de distinguer ce qui résulte d'un processus d'usure de ce qui est dû à un façonnage intentionnel. Ainsi, la surface des petits polissoirs utilisés pour aiguiser des poinçons ou des aiguilles en os et en bois de cervidé présente de profondes rainures dans lesquelles s'insèrent les baguettes à polir, et dont on peut se demander, même s'il ne fait pas de doute qu'un usage répété les a creusées de plus en plus, si elles n'ont pas au moins été amorcées au préalable afin de faciliter et guider le travail de polissage (Fig. 1). De même, des petits récipients en pierre présentent des stries et des traces de poli qui pourraient résulter $\mathrm{du}$ creusement préalable de la cuvette aussi bien que du malaxage de certaines substances dans la concavité. Difficile de dire dans ce cas, si l'on a affaire à un outil simple non façonné - un naturefact - ou à un artefact légèrement aménagé.

Laurence Astruc rappelle que certains auteurs qualifient de «simples» les outils façonnés sommairement mais qui ne servent qu'une fois et se caractérisent par leur caractère «expédient». Il s'agit là de notre deuxième définition de la simplicité. Ces outils sont rudimentaires,

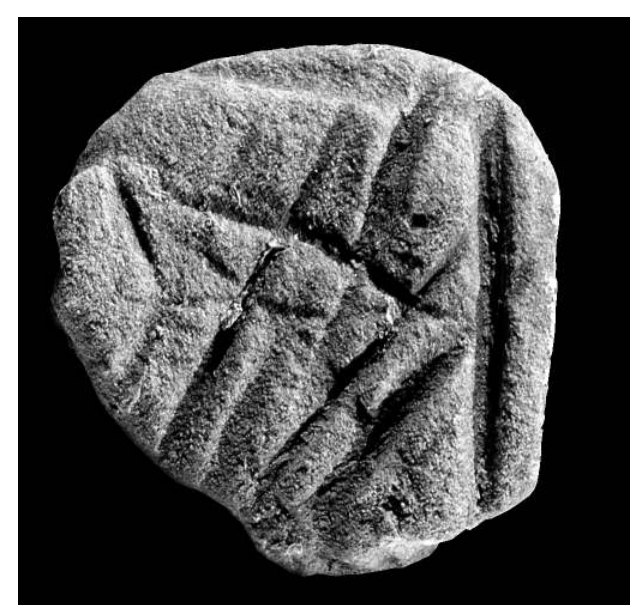

Fig. 1 - Petit bloc de grès présentant des rainures indiquant qu'il a peut-être servi de polissoir à aiguilles. Abri de La Madeleine (Dordogne). Magdalénien. 5,4 ×5 cm. Musée d'archéologie nationale. (C) S. A. de Beaune.

fabriqués sur le moment, utilisés brièvement et rejetés ensuite sans être réutilisés par la suite 6 . Ces outils «expédients» répondent dans ce cas à un besoin immédiat. Ainsi, les plaquettes utilisées comme luminaires dans les grottes et abandonnées sur place après la visite seraient des outils simples, par opposition aux lampes en pierre concaves retrouvées dans les habitats qui ont servi à de multiples reprises (Fig. 2) ${ }^{7}$. Pourtant, un grand nombre de lampes domestiques, qu'on peut supposer avoir été utilisées à plusieurs reprises, ne sont pas plus façonnées que les simples plaquettes retrouvées en grotte. Elles sont simples au premier sens que nous avons donné au mot, mais pas au deuxième. En d'autres termes, un naturefact peut ne pas être un outil expédient. À l'inverse, il y a peu de chance pour qu'un outil expédient soit un artefact: on ne va pas se donner la peine de façonner un outil si $c^{\prime}$ est pour ne l'utiliser qu'une fois (mais 
nous allons tout de même en rencontrer un exemple). Et il n'est pas toujours facile de déterminer quand un outil est expédient: seuls le contexte de sa découverte, la densité et l'intensité des traces d'usage visibles à sa surface ou encore la présence d'éventuels indices de ravivage ou de remploi peuvent permettre de déterminer si l'utilisation a été occasionnelle ou de longue durée. Sans parler de ceux qui, quoique médiocrement façonnés, étaient destinés à un long usage, mais ont été perdus accidentellement après une courte durée d'utilisation. L'archéologue aujourd'hui les voit comme expédients, mais leurs usagers ne les avaient pas conçus comme tels.

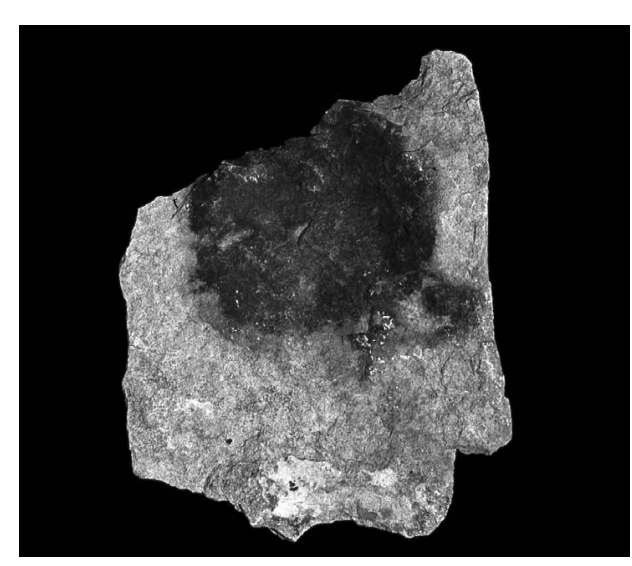

Fig. 2 - Plaquette de calcaire parfaitement plane ayant servi de luminaire d'appoint. Elle présente une plage charbonneuse auréolée d'une frange rubéfiée. Grotte Blanchard, La Garenne (Indre).

Magdalénien. 22,2 × 19,6 × 1,6 cm. Musée archéologique d'Argentomagus (Saint-Marcel, Vienne). (C) S. A. de Beaune.

\section{L'outil est-il le propre de l'homme?}

Les outils simples, en l'un ou l'autre sens, ne sont pas l'apanage des hommes puisque de tels outils sont signalés chez plusieurs espèces animales. La question de l'utilisation d'outils par les animaux ne pose en effet aucun problème dans le cas des naturefacts, tant les exemples de tels usages abondent: pierres pour casser les noix, brindilles pour pêcher les termites, branches ou baguettes pour attraper un fruit dans un arbre ou flottant sur l'eau, feuille pour récolter l'eau de pluie... Les plus célèbres de ces utilisateurs d'outils, les chimpanzés, ne sont pas les seuls animaux à employer à l'occasion des pierres, des brindilles ou des branches comme outils ${ }^{8}$. Certains oiseaux, en particulier les corbeaux calédoniens, sont réputés pour cela. D'autres sont plus inattendus, comme les loutres de mer (Enhydra lutris) qui brisent les coquillages sur leur poitrine avec des galets .

Les cas d'outils bruts légèrement transformés par les animaux sont plus rares, mais on peut citer les baguettes que certains chimpanzés mâchouillent pour en émousser l'extrémité et ainsi faciliter la pêche aux termites. Certains oiseaux pêchent des larves de capricorne à l'aide de brindilles qu'ils semblent choisir en fonction de leur longueur et de leur rigidité ${ }^{10}$. C'est le cas, par exemple, du corbeau calédonien, qui va même jusqu'à créer un crochet ou un hameçon en courbant un fil de fer à l'une de ses extrémités s'il ne dispose pas de rameau ou de brindille adapté ${ }^{11}$. Ces outils sont cependant abandonnés après usage et rentrent donc dans la catégorie des outils 
simples au deuxième sens du mot malgré le léger aménagement qu'ils subissent.
Voilà un artefact qui aura eu un usage expéditif!

\section{Caractéristiques techniques des outils simples}

Tous les matériaux disponibles dans la nature peuvent être utilisés comme outils. La pierre sous forme de galets, de blocs ou de plaquettes a donné des percuteurs, broyeurs, molettes, pierres à moudre, enclumes, billots..., tous outils qui entrent le plus souvent dans la catégorie des naturefacts ${ }^{12}$. De tels outils lithiques sont attestés dès le Paléolithique archaïque, avec les casse-noix et les enclumes de l'Oldowayen; ils sont particulièrement abondants dans certains sites où ils peuvent représenter jusqu'à $50 \%$ du matériel ${ }^{13}$. À partir d'une étude expérimentale récente, Ignacio de la Torre a pu mettre en place une typologie des traces observées en fonction de la tâche accomplie ${ }^{14}$.

Les naturefacts peuvent être aussi en matière dure animale. Des esquilles osseuses ont servi très tôt de poinçons, de maillets, de sortes de percuteurs pour effectuer la retouche de certains outils en pierre taillée (Fig. 3$)^{15}$, voire de récipients comme les cavités cotyloïdes - fragments concaves d'os iliaque - de renne utilisées pour stocker du colorant.

La surface ou la pointe de certaines dents a été employée brute (Fig. 4), les canines de sanglier datées du Mésolithique et converties en poinçons étant sans doute les plus spectaculaires. Les bois de cervidé non travaillés ont servi de pics et de piochons pour extraire les rognons de silex des parois calcaires au Mésolithique et au Néolithique ${ }^{16}$. Les coquillages ont été utilisés comme récipients (Fig. 5) ou comme couteaux surtout par les populations côtières, en particulier dans les îles du Pacifique.

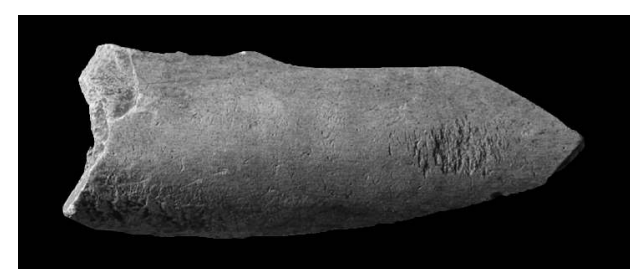

Fig. 3 - Fragment de diaphyse portant des traces d'utilisation interprété comme ayant servi de retouchoir. Grand abri de La Ferrassie (Dordogne). Moustérien. L. env. $11 \mathrm{~cm}$. Musée d'archéologie nationale. (c) S. A. de Beaune.

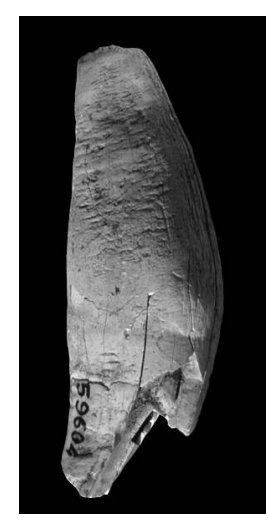

Fig. 4 - Canine de Felis spelaeus portant des traces d'utilisation sur une de ses faces interprétées comme résultant d'un usage en «cousoir». Abri du Ruth (Dordogne). Aurignacien. L. 9,4 ×3,3 cm. Musée d'archéologie nationale. (c) S. A. de Beaune. 


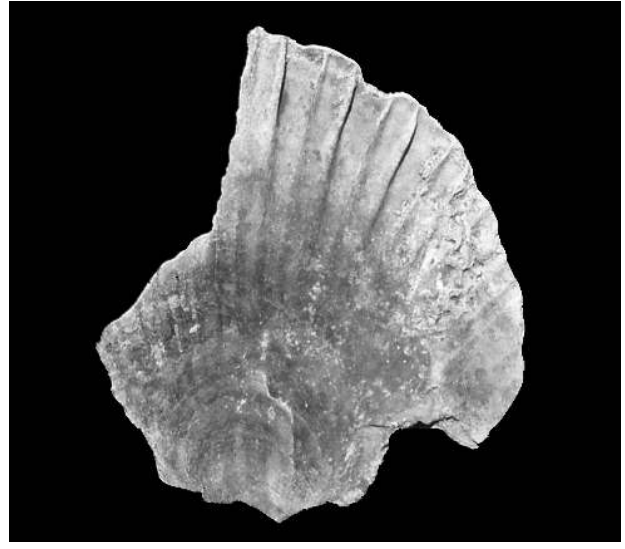

Fig. 5 - Pecten contenant du charbon et de l'ocre dans sa concavité indiquant qu'il a servi de godet à couleur et de lampe. Chapelle de la Lionne, grotte des Trois-Frères (Ariège). Magdalénien. $11 \times 9,7$ cm. Collection Bégouën, Musée de Pujols. (C) S. A. de Beaune.
Les matériaux d'origine végétale comme le bois ont servi de tout temps à de multiples usages sans aucun aménagement. Aujourd'hui encore, des hameçons de fortune sont constitués à partir d'une allumette ou d'une épine blanche. L'écorce des arbres, mais aussi vraisemblablement les feuilles ont servi très tôt de récipient, comme l'atteste la remarquable découverte d'un fragment de plateau en écorce, peut-être utilisé pour la collecte à Kalambo Falls, en Zambie, dans un niveau acheuléen daté d'environ 180000 ans $^{17}$.

\section{Comment reconnaître les outils simples en contexte archéologique?}

Étant donné que les naturefacts se caractérisent par un aménagement faible ou nul, il n'est pas toujours facile de les reconnaître. Deux critères principaux peuvent y aider.

Tout d'abord, ces vestiges ont généralement été apportés par l'homme dans son habitat. Leur origine exogène dans le site est mise en évidence par un géologue ou un pétrographe, s'il s'agit d'objets en pierre, par un archéozoologue, pour les vestiges en matière dure animale - os, ivoire ou bois de cervidé. Il faut cependant garder à l'esprit que des objets apportés dans l'habitat n'ont pas nécessairement servi d'outils, comme les éléments de charpente, les pierres de foyer ou encore les blocs-sièges.
Pour pouvoir affirmer que ces éléments naturels apportés par l'homme sur son lieu de vie ont bien été utilisés, il faut examiner leur surface pour y déceler des traces d'usage. Leur utilisation a provoqué une modification de leur aspect. Selon le travail auquel ils ont été destinés, ils présentent des traces d'impact, des stries, un poli d'usure, voire une véritable altération de leur forme par l'apparition de cupules d'usage, de rainures, de méplats sur leur bord... 


\section{Comment aborder l'étude des outils simples en contexte archéologique?}

Les préhistoriens ont aujourd'hui tendance à se focaliser sur les processus de fabrication des artefacts et négligent bien souvent leur fonction, au motif que les techniques d'élaboration et de fabrication nous renseignent davantage sur les savoir-faire en jeu. Lorsqu'ils ont affaire à des naturefacts et qu'ils décident malgré tout de les étudier - ce qui est rarement le cas -, ils sont évidemment contraints de se montrer attentifs aux procédés d'utilisation de ces objets, vu qu'il n'y a pas grand-chose à dire sur leur fabrication.

L'objectif est alors de retrouver, à partir des traces d'utilisation, les gestes exécutés et, partant, le fonctionnement de l'objet. Le problème se pose moins pour les artefacts car, si les usagers ont pris la peine de les aménager, c'est parce qu'ils comptaient les utiliser d'une certaine manière, sur lequel l'aménagement luimême permet de faire des hypothèses: $\mathrm{si}$ un outil a une arête aiguisée, c'est sans doute parce qu'il servait à couper. Pour les naturefacts, au côté d'autres caractéristiques dont nous parlerons plus loin (forme de l'outil, matière première, etc.), ce sont surtout les traces d'utilisation qui sont en mesure de nous dire comment ils ont été utilisés (leur fonctionnement) et à quoi ils ont servi (leur fonction). Un troisième niveau d'interprétation est celui de l'activité: une fois la fonction et le fonctionnement élucidés, il faut encore se pencher sur la question de l'activité dans laquelle s'insère le maniement de l'outil. Quoique liées, ces distinctions ne se superposent pas exactement: connaître le geste exécuté ne suffit pas toujours à comprendre la fonction de l'outil, et connaître sa fonction ne suffit pas toujours à dire dans quelle activité il a été impliqué18.

$\mathrm{Et}$, pour ce qui est des inférences possibles à partir des traces d'utilisation, les choses se présentent de façon différente selon que l'outil en question est totalement inconnu ou au contraire attesté dans le registre ethnographique ou historique. Dans le premier cas, l'absence d'un quelconque référentiel contraint l'archéologue à élucider l'origine des traces d'utilisation observées à partir de leur comparaison avec des traces obtenues sur des répliques expérimentales et de la prise en compte d'autres données fournies par le contexte archéologique ${ }^{19}$.

Dans le second cas, la fonction de l'objet est supposée grâce à des données comparatives. Ainsi, un bloc, une plaque ou un galet, dont la forme et les traces d'utilisation suggèrent un usage pour moudre, permettra de classer cet objet dans la catégorie des meules, des broyeurs ou des molettes. On a donc d'emblée une idée de la fonction de l'objet, mais pas de son fonctionnement précis ni de la nature des substances animales, végétales ou minérales - qu'il a permis de moudre ou d'écraser, et donc de l'activité à laquelle il a participé. De même, un petit polissoir à main est facilement identifiable grâce aux rainures visibles sur sa surface, mais il est plus difficile de savoir s'il a servi à polir des aiguilles, des pointes de sagaie ou tout autre objet mince et allongé... 


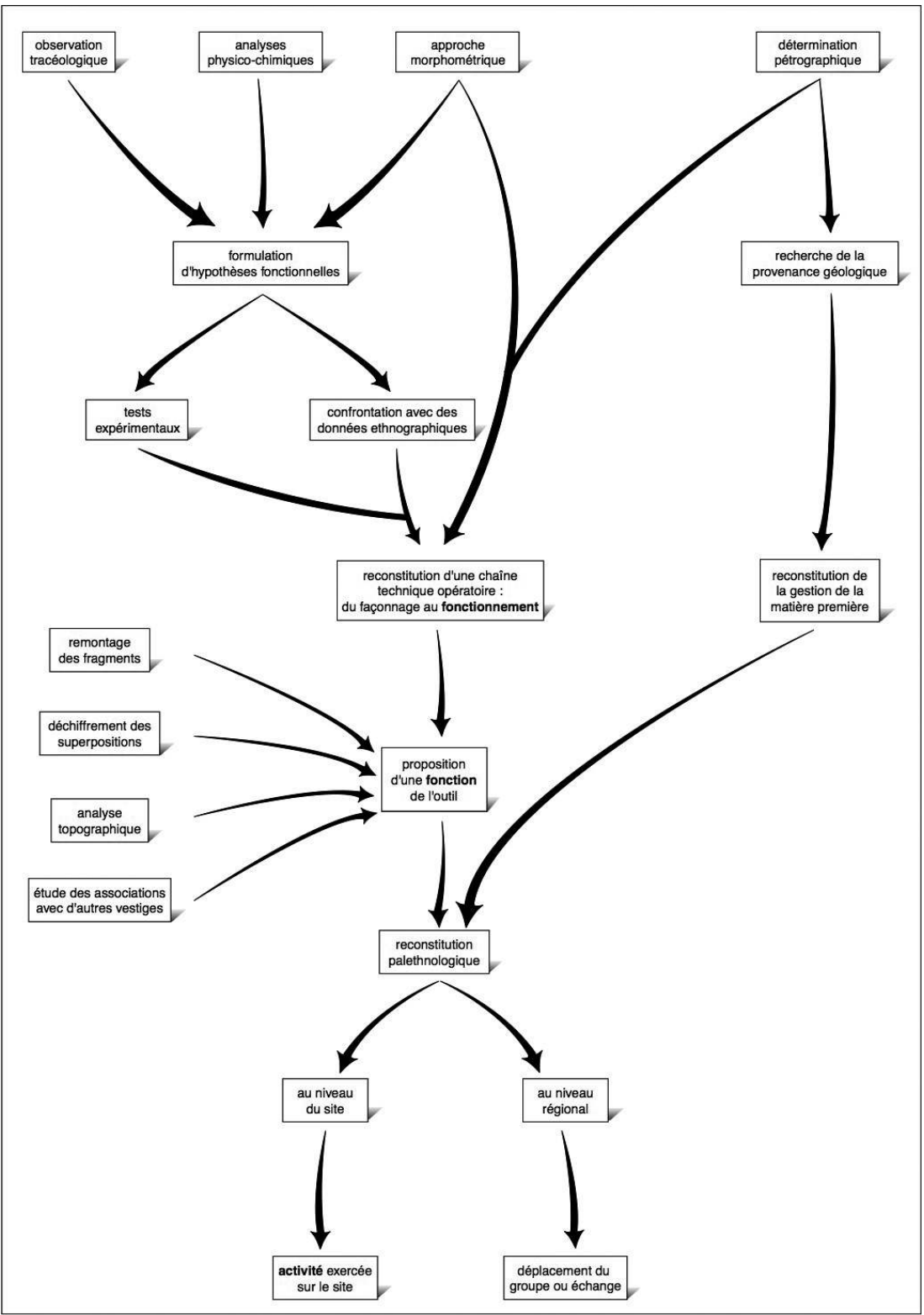

Fig. 6 - Principales étapes méthodologiques nécessaires pour appréhender le fonctionnement et la fonction d'un outil simple, retrouver l'activité dans laquelle il s'insère et le resituer dans son contexte.

Pour tenter d'élucider la fonction, le fonctionnement et l'activité concernée par l'outil, il faut développer des outils diagnostiques par l'étude des traces d'usage.
Selon toute logique, les différentes actions exécutées sur la matière laissent des traces particulières. Ainsi, une percussion lancée punctiforme laisse des traces 
d'impact sur la surface; un polissage exécuté en un mouvement d'avant en arrière produit une surface polie avec éventuellement des stries révélant la direction du geste. À partir de la typologie des percussions mise au point par André LeroiGourhan ${ }^{20}$ et de l'observation d'un grand nombre d'outils simples en pierre, j'ai pu mettre en évidence un certain nombre de variables permettant de reconnaître la spécificité de chaque type d'outil. Outre la nature des traces d'utilisation ellesmêmes et leur emplacement sur l'objet, ces variables concernent la dimension de l'objet, sa forme, sa matière première, son volume, son poids... ${ }^{21}$

L'origine des traces d'utilisation ne peut être comprise par une simple observation et elle doit se doubler de protocoles expérimentaux rigoureux susceptibles d'expliquer les processus de formation des traces. L'expérimentation vise à vérifier la plausibilité de certaines hypothèses et à élaborer des référentiels afin de mieux comprendre le fonctionnement des outils et de comparer les traces obtenues expérimentalement aux traces archéologiques.

Le recours à des données d'ordre ethnographique ou historique est utile, non comme source d'explications, mais comme source d'hypothèses. Puisque les outils subissent les mêmes contraintes de la matière quels que soient le lieu et l'époque considérés, la comparaison d'une technique observée sur un terrain ethnographique à des objets préhistoriques peut apporter des éléments de réponse aussi intéressants, sinon plus, que l'expérimentation. Cette approche comparative peut être étendue aux données historiques.

L'idéal est évidemment de pouvoir mener parallèlement une approche archéologique, expérimentale et ethnographique (Fig. 6). C'est ce j'ai pu faire pour l'étude des lampes en pierre paléolithiques qui constituent un bel exemple d'outil simple, grâce à une approche comparative des divers types de lampes utilisées par les populations inuits. Cela a permis de mieux cerner les contraintes techniques inhérentes à ce type de luminaire ${ }^{22}$.

L'étude du contexte archéologique est fort utile aussi pour comprendre la place de ces outils simples dans le système technique d'un groupe humain. Ainsi, l'étude des meules ayant servi à moudre des grains de céréales sauvages retrouvées sur le site épipaléolithique d'Abu Hureïra (Syrie) a été doublée d'une analyse anthropologique des squelettes retrouvés dans le même site, réalisée par Theya Molleson. Ce qui a permis de mettre en évidence un certain nombre de lésions articulaires, en particulier au niveau des orteils, des chevilles, des genoux et du bas de la colonne vertébrale, lésions qui n'ont pu être provoquées que par la posture prolongée et réitérée adoptée lors de la mouture des grains: ces constatations permettent d'avancer l'hypothèse que les personnes qui pratiquaient la mouture étaient agenouillées et déplaçaient le broyeur sur la meule en un geste répétitif des bras, d'avant en arrière. Cela confirme aussi que la mouture était une activité féminine comme c'est très généralement le $\mathrm{cas}^{23}$.

$\mathrm{Au}$ final, ces outils simples, pourtant souvent négligés lors de l'étude monographique des sites archéologiques, apportent leur lot d'informations, au même titre que les outils plus complexes, et peuvent permettre de mieux 
saisir la nature d'un site donné ou de ses aires d'activité (habitat, halte de chasse, simple bivouac...). Des activités qui resteraient sans cela des activités fantômes peuvent même parfois être mises en évidence. Ainsi, la présence de blocs en pierre ayant servi de meules et de broyeurs signale une activité domestique telle que la préparation de la nourriture; des molettes de corroyage, simples galets fortement lustrés et souvent ocrés, attestent de la préparation de peaux.

\section{Quelques remarques conclusives}

Les outils simples entrent dans une grande quantité d'activités d'acquisition (chasse, pêche), de fabrication (travail de la pierre, de l'os, de l'ivoire...) et de consommation (préparation de la nourriture...). Ils font souvent partie de la panoplie des artisans au même titre que d'autres outils plus élaborés. Alors qu'ils ne nécessitent aucun investissement technique, ces outils simples font ainsi partie intégrante d'une chaîne opératoire qui peut parfois s'avérer complexe. Ainsi, le tailleur de pierre du Paléolithique aura toujours un simple galet dans sa besace, percuteur indispensable au débitage et à la retouche du silex. Que ce soit à Khirokitia, à Chypre, au VII millénaire, ou à Malia, en Crète, au II e millénaire, l'artisan fabriquant les vaisselles en pierre ne dédaigne pas d'utiliser de simples galets bruts en guise de percuteurs ou de bouchardes, qui peuvent éventuellement faire l'objet de ravivages ${ }^{24}$.

Autre constatation qui mérite d'être rappelée: ces outils simples ne sont pas nécessairement anciens. Ils ont parfois perduré à travers les millénaires, comme les maillets et les galets qui servent à concasser des fruits à coquille dure, utilisés encore de nos jours. En revanche, le statut de ces outils simples inventés très anciennement peut évoluer au cours du temps: ils ont tendance à être remplacés par des inventions plus complexes et plus spécialisées, mais ne disparaissent pas pour autant. Ils deviennent alors de simples expédients: qui n'a pas eu recours, un jour ou l'autre, à un galet en guise de casse-noix ou de marteau, faute d'avoir l'outil spécialisé sous la main à ce moment?

$\mathrm{Si}$ on les oppose aux outils "complexes», on note, à la suite de Laurence Astruc, que ces outils simples, non ou peu façonnés, sont généralement moins spécialisés et produits à partir de matières premières locales, alors que les outils complexes, plus spécialisés, sont réalisés sur des matériaux particuliers parfois venus de loin et sont fabriqués selon des méthodes plus élaborées qui supposent un savoir-faire important. Ils résultent souvent de chaînes opératoires dont les différentes étapes peuvent être déconnectées dans le temps et dans l'espace, et sont le fait de spécialistes ${ }^{25}$. Ceci s'explique par le fait que, même si les deux notions ne s'équivalent pas, comme nous l'avons vu, les naturefacts sont souvent des outils expédients.

L'usage de ces outils simples est-il réservé à une certaine classe de la population? En d'autres termes, la simplicité d'un outil peut-elle être liée à son 
statut et à son utilisateur? C'est l'hypothèse avancée par Paola Tabet, qui croit déceler, à partir de la compilation de descriptions d'outillage technique de nombreuses populations de chasseurscueilleurs, mais aussi de groupes horticoles et agricoles, une différence entre outils féminins et outils masculins, pour les mêmes activités. Pour elle, les outils frustes, non travaillés, seraient essentiellement utilisés par les femmes, les hommes se réservant l'accès à des outils plus complexes pour la même activité26. Il est dommage que cette étude fort documentée ait un ton féministe militant qui en limite la portée scientifique. On soupçonne l'auteur d'avoir un peu tiré sur la documentation pour arriver à sa démonstration. Pourtant, la question mérite réflexion et $c^{\prime}$ est précisément pourquoi le séminaire évoqué au début de ce texte traitait d'un second thème outre celui des outils simples -, celui de la répartition des tâches dans les sociétés préindustrielles, en particulier entre les sexes. Il est un fait que, s'il n'y a pas de tâches féminines en elles-mêmes, il arrive souvent qu'une même tâche soit exécutée de manière différente selon qu'elle est confiée à des hommes ou à des femmes. La technique gestuelle peut varier - comme par exemple dans le cas du semis des céréales au XIX ${ }^{\mathrm{e}}$ siècle, que les hommes et les femmes n'exécutaient pas avec le même geste, le semis à la volée étant rigoureusement masculin. Il arrive aussi que ce soit l'outil qui diffère selon l'utilisateur. Lorsqu'hommes et femmes sont tous deux potiers, il est fréquent que les pots soient montés à la motte ou au colombin par les femmes, l'usage du tour étant réservé aux hommes. Cependant, pour les périodes très anciennes qui nous occupent ici, et même s'il est vraisemblable que la division des tâches est apparue très tôt, rien n'autorise à penser - du moins en l'état actuel de la documentation archéologique disponible - qu'il existait une différence entre outils masculins et outils féminins pour les mêmes tâches et que les premiers étaient nécessairement complexes et les seconds simples.

\section{Notes}

1. Professeure à l'université Jean Moulin Lyon 3 et chercheuse à l'UMR Archéologies et Sciences de l'Antiquité, Sophie Archambault de Beaune travaille sur les comportements techniques et les aptitudes cognitives de l'homme préhistorique. Elle a notamment publié Pour une archéologie du geste et L'homme et l'outil (CNRS Éditions, 2000 et 2008) et co-dirigé Cognitive Archaeology and Human Evolution (CUP, 2009). Contact: sophie.de-beaune@mae.cnrs.fr; http://www. mae.u-paris10.fr/arscan/Sophie-Archambaultde-Beaune.html.

2. François Sigaut (1940-2012), directeur d'études à l'École des hautes études en sciences sociales (EHESS), était spécialiste de l'histoire des techniques, en particulier des techniques agricoles. Pour une biographie de François Sigaut, je me permets de renvoyer à Sophie A. de Beaune, "François Sigaut (1940-2012), agronome et technologue», Revue de synthèse, t. 134, $6^{\mathrm{e}}$ série, $\mathrm{n}^{\circ}$ 1, 2013, p. 115124. Haris Procopiou, maître de conférences à l'université Paris I Panthéon-Sorbonne, est spécialiste de la protohistoire égéenne et s'intéresse tout spécialement aux techniques du travail de la pierre. Les séminaires, qui se poursuivent encore aujourd'hui malgré la disparition de François Sigaut, ont traité la question de l'outil simple en particulier lors de séances qui se sont déroulées de 2007 à 2010 (voir comptes rendus en ligne: http://www.ehess.fr/ fr/enseignement/enseignements/).

3. Wendell H. Oswalt, An anthropological analysis of food-getting technology, Hoboken (NJ), John Wiley \& Sons, 1976.

4. Narjys El Alaoui, "Une presse à huile au Maroc», Techniques \& Culture, $\mathrm{n}^{\circ}$ 48-49, 2007, p. 189-218.

5. Sophie A. de Beaune, "Origine du matériel de mouture: innovation et continuité du Paléolithique au Néolithique», dans Mouette Barboff, 
François Sigaut, Cozette Griffin-Kremer et Robert Kremer (dir.), Meules à grains, Actes du colloque international "Extraction, façonnage, commerce et utilisation des meules de moulin - Une industrie dans la longue durée», 16-19 mai 2002, La Ferté-sousJouarre/Paris, Ibis Press/Maison des Sciences de l'Homme, 2003, p. 15-30.

6. Laurence Astruc, ce numéro; Lewis Binford, «Forty-seven trips: A case study in the character of archaeological formation process", dans Richard V. S. Wright (dir.), Stone tools as cultural markers, Canberra, Australian Institute of Aboriginal Studies, 1977, p. 24-36.

7. Sophie A. de Beaune, «Palaeolithic lamps and their specialization. A hypothesis", Current Anthropology, vol. $28 \mathrm{n}^{\circ} 4,1987$, p. 569-577.

8. Pour les outils des chimpanzés, voir par exemple Christophe Boesch et Hedwige Boesch-Achermann, The Chimpanzees of the Taï Forest: behavioural ecology and evolution, Oxford, Oxford University Press, 2000.

9. K.R.L. Hall et George B. Schaller, «Toolusing behaviour of the California sea otter», Journal of Mammalogy, vol. 45 n², 1964, p. 287-298.

10. Voir, par exemple, les observations réalisées à l'aide de mini-caméras dotées d'un émetteur radio VHF fixées sous les grandes plumes de la queue de corbeaux calédoniens (Lucas A. Bluff et al., "Tool use by wild New Caledonian Corvus moneduloides at natural foraging sites", Proceedings of the Royal Society, B., 2009, doi:10.1098/ rspb.2009.1953).

11. Voir Gavin R. Hunt et Russell D. Gray, "Diversification and cumulative evolution in New Caledonian crow tool manufacture», Proceedings of the Royal Society, B 270, 2003, p. 867-874; Alex A. S. Weir, Jackie Chappell et Alex Kacelnik, "Shaping of hooks in New Caledonian crows", Science, $\mathrm{n}^{\circ} 297,2002$, p. 981.

12. Sophie A. de Beaune, Pour une archéologie du geste. Broyer, moudre, piler, des premiers chasseurs aux premiers agriculteurs, Paris, CNRS Éditions, 2000.

13. Voir, entre autres, Jean Chavaillon, «Essai pour une typologie du matériel de percussion», Bull. Soc. préhist. franç., t. 76, n 8, 1979, p. 230-233; Mary D. Leakey, dans Mary D. Leakey et D. A. Roe (dir.), Olduvai Gorge, vol. 5. Excavations in Beds III and IV and the Masek Beds, 1968-1971, Cambridge, Cambridge University Press, 1994, p. 15-129.

14. Ignacio de la Torre et al., "Experimental protocols fort the study of battered stone anvils from Olduvai Gorge (Tanzania)», Journal of Archaeological Science, 40, 2013, p. 313-332.
15. Élise Tartar, «L'équipement en os: une fenêtre sur le quotidien des Paléolithiques", dans Sophie A. de Beaune (dir.), Chasseurs-cueilleurs. Comment vivaient nos ancêtres du Paléolithique supérieur, Paris, CNRS Éditions, 2013 [2007], p. 59-68; Sophie A. de Beaune, Les galets utilisés au Paléolithique supérieur. Approche archéologique et expérimentale, Paris, CNRS Éditions, 1997, annexe p. 169-192.

16. Voir, par exemple, l'outillage minier en bois de cerf du site de Jablines, dans Françoise Bostyn et Yves Lanchon (dir.), Jablines, Le Haut Château (Seine-et-Marne). Une minière de silex au Néolithique, Paris, Maison des Sciences de l'Homme, Documents d'archéologie française, $n^{\circ} 35,1992$, p. 102120.

17. J. Desmond Clark, Kalambo Falls. Prehistoric site, vol. III, The Earlier Cultures: Middle and Earlier Stone Age, Cambridge, Cambridge University Press, p. 318 et 324.

18. Sur ces questions terminologiques, voir François Sigaut, Comment Homo devint faber. Comment l'outil fit l'homme, Paris, CNRS Éditions, coll. Le passé recomposé, 2012, p. 53 sq., et Sophie A. de Beaune, 2000, op. cit., p. 38 sq.

19. Pour un exemple d'une telle étude, voir Sophie A. de Beaune, 1997, op. cit.

20. André Leroi-Gourhan, L'homme et la matière, Paris, Albin Michel, 1943.

21. Le travail d'ensemble dont est tiré cet article est présenté dans Sophie A. de Beaune, 2000, op. cit.

22. Sophie A. de Beaune, Lampes et godets au Paléolithique, Paris, CNRS Éditions, 1987.

23. Theya Molleson, "The eloquent bones of Abu Hureyra", Scientific American, août 1994, p. 60-65.

24. Élise Morero et Hara Procopiou, «Des outils simples pour des artisans spécialisés: l'exemple du Quartier Mu de Malia», dans Laurence Astruc et al. (dir.), Normes techniques et pratiques sociales. De la simplicité des outillages pré- et protohistoriques, Antibes, APDCA, 2006, p. 383-390, et Laurence Astruc, ce numéro.

25. Voir ce numéro, Laurence Astruc.

26. Paola Tabet, "Les mains, les outils, les armes », L'Homme, vol. 19, n³-4, 1979, p. 5-61. 